University of Nebraska - Lincoln

DigitalCommons@University of Nebraska - Lincoln

10-15-1974

\title{
Deduction of asymptotic Steinmann relations from the Regge hypothesis
}

\author{
Paul Finkler \\ University of Nebraska-Lincoln, pfinkler1@unl.edu \\ C. Edward Jones \\ University of Nebraska-Lincoln \\ M. Misheloff \\ University of Nebraska-Lincoln
}

Follow this and additional works at: https://digitalcommons.unl.edu/physicsfinkler

Part of the Physics Commons

Finkler, Paul; Jones, C. Edward; and Misheloff, M., "Deduction of asymptotic Steinmann relations from the Regge hypothesis" (1974). Paul Finkler Papers. 10.

https://digitalcommons.unl.edu/physicsfinkler/10

This Article is brought to you for free and open access by the Research Papers in Physics and Astronomy at DigitalCommons@University of Nebraska - Lincoln. It has been accepted for inclusion in Paul Finkler Papers by an authorized administrator of DigitalCommons@University of Nebraska - Lincoln. 


\title{
Deduction of asymptotic Steinmann relations from the Regge hypothesis
}

\author{
P. Finkler, C. E. Jones, and M. N. Misheloff \\ Behlen Laboratory of Physics, University of Nebraska, Lincoln, Nebraska 68508
}

(Received 31 December 1973)

\begin{abstract}
The analytic structure of the double-Regge vertex, which has previously been obtained from the Steinmann relation, is derived by using a natural generalization of the Regge hypothesis.
\end{abstract}

\section{INTRODUCTION}

The general requirements of Steinmann ${ }^{1}$ on the discontinuities of many-particle scattering amplitudes have been recently applied to asymptotic Regge terms. This has resulted in restrictions on the analytic structure of such Regge terms. ${ }^{2}$ We suggest here that a very natural extension of the basic Regge hypothesis itself makes the Steinmann structure for Regge terms mandatory. This extension of the Regge hypothesis assumes a Lehmann-type neighborhood of analyticity for general group-theory angles. This same assumption has already been applied elsewhere to derive properties of the asymptotic six-point function. ${ }^{3}$ However, its relation to the Steinmann question has not been explored before.

The point of view which we adopt in this paper is that the group-theory variables, rather than the invariant variables, are the fundamental ones for expressing Regge asymptotic behavior. As we show, this approach can lead to the appearance of several terms in a given Regge asymptotic limit. The principle by which we include these distinct terms is important and needs some explanation.

A key feature of our procedure is the introduction of extra group-theory variables so that the number of such variables exceeds the actual number of invariant variables. The extra group-theory variables specify how one of the Lorentz frames in the problem is chosen. This arbitrariness in the selection of the Lorentz frame we refer to as a "gauge" freedom. Since the scattering amplitude, which is a Lorentz invariant, cannot depend upon how this particular Lorentz frame was picked, a change in the group variables resulting from a change of frame (called a "gauge transformation") must leave the amplitude invariant. This invariance of the amplitude under a gauge transformation of the group variables shows that the amplitude is not an independent function of the extra group variables. It also shows that the use of different values of the group variables-which are connected by a gauge transformation-must lead to the same value (in our case, asymptotic limit) for the amplitude.

Our basic assumption is that the Regge asymptotic limit may be taken in the group variables in any way that leads to the desired limit for the invariant variables and that Lehmann-type analyticity exists in this limit in the group-theory angles. Thus a gauge transformation on the grouptheory variables in the Regge asymptotic limit gives an equivalent description of the limit with the same analytic properties and with nothing new added.

However, in certain special limits, we show that the same limit in the invariant variables can be achieved by different group-theory variables which are not related by a gauge transformation. The absence of a gauge transformation relating the variables in these special limits is underscored by the different analytic properties of the amplitude when approaching such limits. Since we regard the group variables as fundamental, we must take each of the distinct (i.e., unrelated by a gauge transformation) ways of approaching the limit as valid and, hence, include each term in the asymptotic behavior. Thus we find within the grouptheory discussion a natural principle for adding, in some cases, several Regge terms in the asymptotic limit. In the problem we consider, this set of terms is just that required by the Steinmann relations.

The ability to regard Steinmann relations as contained within the basic Regge structure may have useful advantages. Our results illustrate once again how tidily the assumption of Regge behavior meshes with certain over-all conditions that scattering amplitudes must obey. In this connection we point out how a potential conflict between Regge behavior and the Steinmann relations is avoided in one case by the special kinematic character of the Regge limit.

We consider here the five-point function in the double-Regge limit; however, the approach can undoubtedly be applied more generally. The subject of signature, which seems to be a separate issue altogether, is not introduced. 


\section{REVIEW OF DOUBLE-REGGE LIMIT}

In this section we present a brief review of the standard treatment of the double-Regge limit for the scattering amplitude of five physical spinless particles. In the next section we will consider the double-Regge limit in the case where one of the particle's momentum is spacelike; the standard treatment can be used for that case with only minor modifications.

Multi-Regge limits of scattering amplitudes are most naturally derived when the amplitudes are expressed in terms of the squares of momentum transfers and group variables. ${ }^{4}$ The group variables express the dependence of the amplitude upon transformations between body-fixed Lorentz frames, i.e., frames in which subsets of the external momenta and momentum transfers have specified values which are functions of the external masses and momentum transfers in the subset. ${ }^{4}$ Multi-Regge limits give asymptotic expressions for the amplitude when several transformations between the body-fixed frames are large.

We consider the reaction $P_{1}+P_{2}-P_{3}+P_{4}+P_{5}$ (see Fig. 1). We define the usual invariant variables:

$$
\begin{aligned}
& s=\left(P_{1}+P_{2}\right)^{2}, \\
& t_{1}=\left(P_{1}-P_{3}\right)^{2}, \\
& t_{2}=\left(P_{2}-P_{4}\right)^{2}, \\
& s_{1}=\left(P_{3}+P_{5}\right)^{2}, \\
& s_{2}=\left(P_{4}+P_{5}\right)^{2} .
\end{aligned}
$$

We also define the following four body-fixed frames:

Frame I. The four-momentum transfer $Q_{1} \equiv P_{1}$ $-P_{3}$ has a positive $z$ component; the other components of $Q_{1}$ vanish. The $x$ and $y$ components of $P_{1}$ are zero.

Frame II. $Q_{1}$ has only a positive $z$ component. The $x$ and $y$ components of $P_{5}$ vanish.

Frame III. This is the frame in which $Q_{2} \equiv P_{2}$ $-P_{4}$ has only a positive $z$ component and which is related to frame II by a boost in the $z$ direction. The boost parameter is entirely determined by $t_{1}$, $t_{2}$, and $m_{5}^{2} \equiv P_{5}^{2}$.

Frame IV. The momentum transfer $Q_{2} \equiv P_{2}-P_{4}$ has only a positive $z$ component. The $x$ and $y$ components of $P_{2}$ vanish.

We note that each of frames I, II, and IV are defined only up to an arbitrary $z$ rotation. This fact enables us to express the amplitude, which

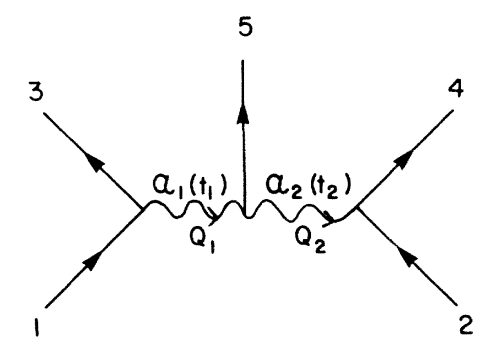

FIG. 1. Double-Regge diagram.

is a function of five independent variables, as a "gauge-invariant"3.5 function of a larger number of variables.

The transformation which takes one from frame I to frame II must be an element of the group $\mathrm{O}(2,1)$, the little group of the vector $Q_{1}$. We denote this $O(2,1)$ element by $g_{1}$. Similarly, the transformation from frame IV to frame III must be an $O(2,1)$ element of the little group of $Q_{2}$ which we will denote by $g_{2}$. The little group elements can be parameterized by

$$
g_{i}=R_{z}\left(\phi_{i}\right) B_{x}\left(\xi_{i}\right) R_{z}\left(\psi_{i}\right) \quad(i=1,2),
$$

where $R_{z}$ denotes a rotation about the $z$ axis and $B_{x}$ denotes a boost along the $x$ axis.

The amplitude can be expressed as a function of $t_{1}, t_{2}, g_{1}$, and $g_{2}$. The gauge invariance of the amplitude alluded to above can now be easily understood. A redefinition of frame II by an arbitrary angle $\chi$ leaves the value of the amplitude unchanged but transforms the variables $\phi_{i}$ into $\phi_{i}+\chi$. This freedom of gauge implies that we can set either $\phi_{1}$ or $\phi_{2}$, but not both, equal to an arbitrary value. Alternatively, the gauge freedom in this case implies that the amplitude depends upon $\phi_{1}$ and $\phi_{2}$ only in the combination $\omega \equiv \phi_{1}-\phi_{2}$. Similar reasoning implies that the amplitude cannot depend upon $\psi_{1}$ or $\psi_{2}$, assuming the external particles are spinless.

The double-Regge region is obtained by allowing $\xi_{1}$ and $\xi_{2}$ to become asymptotic, while the other variables are held fixed. Assuming that the leading singularities in the angular momentum plane are poles, the leading term of the five-point amplitude in this region is given by

$$
\begin{aligned}
T_{5} \underset{\xi_{1}, \xi_{2} \rightarrow \infty}{\sim} & \beta_{1}\left(t_{1}\right) \beta_{2}\left(t_{2}\right)\left(\cosh \xi_{1}\right)^{\alpha_{1}\left(t_{1}\right)}\left(\cosh \xi_{2}\right)^{\alpha_{2}\left(t_{2}\right)} \\
& \times F\left(t_{1}, t_{2}, \omega\right) .
\end{aligned}
$$

In this expression $\beta_{1}$ and $\beta_{2}$ are the standard Regge residue functions for each end of the diagram in Fig. 1 and $F$ describes the double-Regge vertex at 
the center.

Equation (2.3) can be cast in a more familiar form by expressing it in terms of $t_{1}, t_{2}, s_{1}, s_{2}$, and $k \equiv s_{1} s_{2} / s$. To do this we note the following asymptotic relations between these variables and the group-theoretical variables ${ }^{4}$ :

$$
\begin{aligned}
& s_{1} \sim \lambda^{1 / 2}\left(t_{1}, t_{2},{m_{5}}^{2}\right) \lambda^{1 / 2}\left(t_{1},{m_{1}}^{2}, m_{3}{ }^{2}\right) \cosh \xi_{1} /\left(-2 t_{1}\right), \\
& s_{2} \sim \lambda^{1 / 2}\left(t_{1}, t_{2},{m_{5}}^{2}\right) \lambda^{1 / 2}\left(t_{2},{m_{2}}^{2}, m_{4}{ }^{2}\right) \cosh \xi_{2} /\left(-2 t_{2}\right),
\end{aligned}
$$

$\kappa \sim \lambda\left(t_{1}, t_{2}, m_{5}^{2}\right) /\left[2\left(-t_{1}\right)^{1 / 2}\left(-t_{2}\right)^{1 / 2}(\cosh q-\cos \omega)\right]$,

where

$$
\cosh q=\left(m_{5}^{2}-t_{1}-t_{2}\right) /\left[2\left(-t_{1}\right)^{1 / 2}\left(-t_{2}\right)^{1 / 2}\right]
$$

and

$$
\lambda(a, b, c)=a^{2}+b^{2}+c^{2}-2 a b-2 a c-2 b c .
$$

In the physical region the several $\lambda$ functions appearing in (2.4) are all positive. Equation (2.3) now becomes

$$
T_{5} \underset{\substack{s_{1} s_{2} \rightarrow \infty \\ \text { K fixed }}}{\sim} \beta_{1}\left(t_{1}\right) \beta_{2}\left(t_{2}\right)\left(-s_{1}\right)^{\alpha_{1}\left(t_{1}\right)}\left(-s_{2}\right)^{\alpha_{2}\left(t_{2}\right)} V\left(t_{1}, t_{2}, \kappa\right),
$$

where we have absorbed some $t$-dependent factors into the definition of the double-Regge vertex $V\left(t_{1}, t_{2}, \kappa\right)$. Henceforth, we will refer to $V\left(t_{1}, t_{2}, \kappa\right)$ as given by (2.7) as the double-Regge vertex.

\section{DERIVATION OF STEINMANN RELATIONS}

In this section, we show that a natural extension of the Regge hypothesis implies the Steinmann analytic structure ${ }^{1}$ for the five-line amplitude in the double-Regge limit. We shall assume that the double-Regge vertex in Eq. (2.7) can only possess singularities at $k=0$ and $k=\infty .^{2}$ If this were not the case the double-Regge limit would contain spurious asymptotic singularities (e.g., there would be singularities in $s$ whose location would depend upon $s_{1}$ and $s_{2}$ ).

The Steinmann relation ${ }^{1}$ prohibits simultaneous discontinuities in overlapping channel invariants in the physical region; this implies that the doubleRegge vertex can be written in the form ${ }^{2}$

$$
\begin{aligned}
V\left(t_{1}, t_{2}, \kappa\right)= & \kappa^{-\alpha_{1}\left(t_{1}\right)} V_{1}\left(t_{1}, t_{2}, \kappa\right) \\
& +\kappa^{-\alpha_{2}\left(t_{2}\right)} V_{2}\left(t_{1}, t_{2}, \kappa\right),
\end{aligned}
$$

where $V_{1}$ and $V_{2}$ are entire functions of $\kappa$. Now $T_{5}$ in the double-Regge limit becomes ${ }^{2,6}$

$$
\begin{aligned}
T_{5} \underset{\substack{s_{1}, s_{2} \rightarrow \infty \\
\kappa \text { fixed }}}{\sim} \beta_{1} \beta_{2}\left[\left(\frac{s}{s_{2}}\right)^{\alpha}\left(s_{2}\right)^{\alpha} V_{1}\left(t_{1}, t_{2}, \kappa\right)\right. \\
\left.+\left(s_{1}\right)^{\alpha}\left(\frac{s}{s_{1}}\right)^{\alpha_{2}} V_{2}\left(t_{1}, t_{2}, \kappa\right)\right] .
\end{aligned}
$$

The required Steinmann structure is apparent from (3.2); for example, the first term has a discontinuity in $s_{2}$ but none in $s_{1}$ if $V_{1}$ is an entire function of $k$. The essential feature of the Steinmann condition on the double-Regge vertex expressed in Eq. $(3.1)$ is that it gives the singularity structure of $V$ at $\kappa$ equal to zero. We now show that this singularity structure follows naturally from an extension of the Regge hypothesis. Our assumption is that the amplitude, and specifically the asymptotic form of the amplitude is an analytic function of the group-theory angles in a region which includes the physical region. We also assume that a term must be included in the asymptotic behavior for each of the distinct (i.e., unrelated by any gauge transformation) ways of approaching the asymptotic limit by means of group variables. This assumption seems to be a natural generaliza tion of the analyticity of the elastic scattering amplitude in the Lehmann ellipse.

Reference to Eq. (2.4) shows that the neighborhood of $\kappa$ equal to zero is not in the physical region for the scattering of physical particles. Therefore, in order to apply our assumption we will consider the case in which particle 5 has a variable mass and analytically continue in the mass; direct physical meaning can be assigned to such considerations by realizing that a five-line amplitude, in which one particle has a variable mass, is a factor of the residue at a Regge pole in the six-line amplitude.

Specifically, we consider the five-particle scattering amplitude in the region of $m_{5}{ }^{2}$ for which $\lambda\left(t_{1}, t_{2}, m_{5}{ }^{2}\right)<0 .{ }^{5}$ The Regge analysis for this case is very similar to that of physical particle scattering. We mention only the modifications.

In frame $I Q_{1}$ has only a positive $z$ component and only the $x$ and $z$ components of $P_{5}$ are nonvanishing. Frame III is the frame in which $Q_{2}$ has only a positive $z$ component and which is related to frame $I$ by a rotation about the $y$ axis. Frame II is now defined only up to an arbitrary $y$ boost. A redefinition of frame $\Pi$ corresponding to a boost $B_{y}(\eta)$ leads to a complicated gauge transformation of the variables. We may use the gauge freedom to set either $\phi_{1}$ or $\phi_{2}$ but not both equal to zero (or $\pi) .^{3}$ The rest of the analysis in terms of the group variables proceeds as in Sec. II. The final result for the asymptotic behavior of the ampli- 
tude in the gauge $\phi_{1}=0$ is given by

$$
\begin{aligned}
T_{5} \underset{\xi_{1}, \xi_{2} \rightarrow \infty}{\sim} \beta_{1}\left(t_{1}\right) \beta_{2}\left(t_{2}\right)\left(\cosh \xi_{1}\right)^{\alpha_{1}\left(t_{1}\right)}\left(\cosh \xi_{2}\right)^{\alpha_{2}\left(t_{2}\right)} \\
\times F\left(t_{1}, t_{2}, \phi_{2} ; m_{5}^{2}\right) .
\end{aligned}
$$

According to our assumption $F$ is an analytic func- tion of $\phi_{2}$ in a region which includes the real line from 0 to $2 \pi$.

We can express this result in terms of the variables $t_{1}, t_{2}, s_{1}, s_{2}$, and $\kappa$ by using the following asymptotic relationships between these variables and the group-theoretical variables:

$$
\begin{aligned}
& s_{1} \sim \lambda^{1 / 2}\left(t_{1},{m_{1}}^{2},{m_{3}}^{2}\right)\left[-\lambda\left(t_{1}, t_{2},{m_{5}}^{2}\right)\right]^{1 / 2} \sinh \xi_{1} /\left(-2 t_{1}\right), \\
& s_{2} \sim \lambda^{1 / 2}\left(t_{2},{m_{2}}^{2},{m_{4}}^{2}\right)\left[-\lambda\left(t_{1}, t_{2},{m_{5}}^{2}\right)\right]^{1 / 2} \sinh \xi_{2} \cos \phi_{2} /\left(-2 t_{2}\right), \\
& \kappa \sim-\lambda\left(t_{1}, t_{2},{m_{5}}^{2}\right) \cos \phi_{2} /\left[2\left(-t_{1}\right)^{1 / 2}\left(-t_{2}\right)^{1 / 2}\left(1-\cos \phi_{2} \cos \theta\right)\right],
\end{aligned}
$$

where

$$
\cos \theta=\frac{t_{1}+t_{2}-m_{5}^{2}}{2\left(-t_{1}\right)^{1 / 2}\left(-t_{2}\right)^{1 / 2}} .
$$

We note that by letting $\xi_{1} \rightarrow+\infty, \xi_{2} \rightarrow+\infty$, and taking the limit

$$
\phi_{2}-\frac{\pi}{2}-\frac{a}{\left(\sinh \xi_{2}\right)^{\delta}} \quad 0<\delta<1,
$$

we can reach the Regge region $s_{1} \rightarrow \infty, s_{2} \rightarrow \infty, \kappa \rightarrow 0$ as a physical Regge limit. Using (3.4) in the limit (3.6) we can write Eq. (3.3) in the form

$$
\begin{aligned}
T_{5 s_{1}, s_{2} \rightarrow \infty} & \beta_{1}\left(t_{1}\right) \beta_{2}\left(t_{2}\right)\left(-s_{1}\right)^{\alpha_{1}\left(t_{1}\right)}\left(-s_{2}\right)^{\alpha_{2}\left(t_{2}\right)} \kappa^{-\alpha_{2}\left(t_{2}\right)} \\
& \times V_{2}\left(t_{1}, t_{2}, \kappa ; m_{5}^{2}\right)
\end{aligned}
$$

where $V_{2}\left(t_{1}, t_{2}, \kappa ; m_{5}{ }^{2}\right)$ is analytic in the neighborhood of $\kappa$ equal to zero.

It is clear that there is another contribution to the limit $s_{1} \rightarrow \infty, s_{2} \rightarrow \infty$, and $\kappa \rightarrow 0$ which is identical to Eq. (3.7), with the indices 1 and 2 interchanged; this term comes from the limit $\xi_{1} \rightarrow \infty, \xi_{2} \rightarrow \infty$, $\phi_{1} \rightarrow \frac{1}{2} \pi-b /\left(\sinh \xi_{1}\right)^{\delta}$ in the gauge $\phi_{2}=0$. This contribution must be regarded as distinct from the first term because the limit $\phi_{2} \rightarrow \frac{1}{2} \pi$ in the gauge $\phi_{1}=0$ and the limit $\phi_{1}-\frac{1}{2} \pi$ in the gauge $\phi_{2}=0$ cannot be connected by any gauge transformation, despite the fact that they both map into the same limit in the invariant variables. The easiest way to understand this remarkable kinematic fact is to parameterize the little-group elements by

$$
g_{i}=R_{z}\left(\gamma_{i}\right) B_{x}\left(\eta_{i}\right) B_{y}\left(\chi_{i}\right) .
$$

The gauge-invariance principle can be expressed by the statement that the amplitude can depend upon $\chi_{1}$ and $\chi_{2}$ only in the combination $\delta \equiv \chi_{1}-\chi_{2}$. The limit (3.6) in terms of the new parameterization is given by $\eta_{1} \rightarrow+\infty, \eta_{2} \rightarrow+\infty, \delta \rightarrow+\infty$, whereas the limit corresponding to the second term is given by $\eta_{1} \rightarrow+\infty ; \eta_{2} \rightarrow+\infty, \delta \rightarrow-\infty$. Since no gauge transformation will change $\delta$, these are obviously distinct limits. According to our approach each of the two above contributions must be included additively. ${ }^{7}$

The fact that two different Regge terms can contribute to the same region in the invariant variables has been exploited previously by Jones, Low, and Young $^{3}$ and by Abarbanel and Schwimmer ${ }^{8}$ in connection with the asymptotic behavior of the sixline amplitude.

To summarize, for the case in which $\lambda\left(t_{1}, t_{2}, m_{5}{ }^{2}\right)$ $<0$ the asymptotic behavior of the amplitude in the limit $s_{1}, s_{2} \rightarrow \infty$ with the other variables fixed is given by

$$
T_{5}-\beta_{1}\left(t_{1}\right) \beta_{2}\left(t_{2}\right) s_{1}^{\alpha_{1}\left(t_{1}\right)} s_{2}{ }^{\alpha_{2}\left(t_{2}\right)}\left[\kappa^{-\alpha_{1}\left(t_{1}\right)} V_{1}\left(t_{1}, t_{2}, \kappa ; m_{5}{ }^{2}\right)+\kappa^{-\alpha_{2}\left(t_{2}\right)} V_{2}\left(t_{1}, t_{2}, \kappa ; m_{5}{ }^{2}\right)\right],
$$

where $V_{1}$ and $V_{2}$ are entire functions of $\kappa$. The term in brackets in Eq. (3.8) can be identified with the double-Regge vertex defined by Eq. (2.7). This result can now be analytically continued in $m_{5}{ }^{2}$ to points which correspond to physical particle masses. Since the singularity structure in $\kappa$ is independent of $m_{5}{ }^{2}$ over a range of $m_{5}{ }^{2}$, this singularity structure must be independent of $m_{5}{ }^{2}$ for all $m_{5}{ }^{2}$. We thus obtain the analyticity structure of the double-Regge vertex which has previously been derived by using the Steinmann relations.

\section{DISCUSSION}

We have shown that analyticity in group-theory angles leads to a structure for the double-Regge limit which has heretofore been deduced only by employing the Steinmann relations. We would like 
here to make more precise the analogy with Lehmann-type analyticity. The general double $\mathrm{O}(2,1)$ expansion for $T_{5}$ may be written ${ }^{4}$

$$
\begin{aligned}
& T_{5}\left(t_{1}, t_{2}, \xi_{1}, \xi_{2}, \phi\right)=\sum_{m}^{\infty} e^{i m \phi} T_{5 m}\left(t_{1}, t_{2}, \xi_{1}, \xi_{2}\right), \\
& T_{5 m}=\int d l_{1} d l_{2} T_{5 m}^{l_{1} l_{2}}\left(t_{1}, t_{2}\right) d_{0 m}^{l_{1}}\left(\xi_{1}\right) d_{m 0}^{l_{2}}\left(\xi_{2}\right)
\end{aligned}
$$

Our requirement of analyticity in group angles assumes that the series expansion (4.1) exists in some neighborhood of the region $0 \leqslant \phi \leqslant 2 \pi$. Alternatively, one may regard $\mathrm{Eq}$. (4.1) as a Laurent expansion in the variable $z=e^{i \phi}$. In terms of $z$, the analyticity assumption is that $T_{5}$ be analytic in a small annulus about $|z|=1$. This is analogous to the Lehmann-ellipse analyticity of the Legendre series expansion in terms of the $P_{l}(z)$. By contrast the expansion in terms of variables $\xi_{1}$ and $\xi_{2}$ in Eq. (4.2) is not a series expansion but a continuous sum over $l_{1}$ and $l_{2}$; this expansion exists even in the presence of singularities in the subenergies and can be made the basis for an asymp- totic series which is, of course, not convergent.

We now turn to a discussion of the functions $V_{1}$ and $V_{2}$ of Eq. (3.1). As mentioned in Sec. III, $V_{1}$ and $V_{2}$ must be entire functions of $\kappa$. Thus either $V_{1}$ and $V_{2}$ are independent of $\kappa$ or they are singular at $\kappa=\infty$. As discussed by DeTar and Weis ${ }^{2} V_{1}$ and $V_{2}$ cannot be constant if there are particles lying on the trajectories $\alpha_{1}$ and $\alpha_{2}$ with spin greater than zero. Thus presumably $V_{1}$ and $V_{2}$ have essential singularities at $\kappa=\infty$, since values of $t_{1}$ and $t_{2}$ exist with $\alpha_{1}$ and $\alpha_{2}$ equal to integers of arbitrary size. This could lead to an inconsistency with Regge behavior if it were possible to reach $\kappa=\infty$ in the double-Regge limit in the same manner in which the $\kappa=0$ limit was achieved in Sec. III. The inconsistency would arise because a Regge expansion in the physical region of the type we are considering in Sec. III must always yield an asymptotic power behavior in $\kappa$. However, an essential singularity can never be so represented. A systematic study of all limits that can be reached using the techniques of Sec. III reveals that the $\kappa=\infty$ limit can never be achieved in this way; thus the inconsistency is avoided.
${ }^{1}$ O. Steinmann, Helv. Phys. Acta 33, 257 (1960); 33, 347 (1960). See also H. Araki, J. Math. Phys. 2,163 (1961); H. P. Stapp, Phys. Rev. D $\underline{3}, 3177$ (1971).

${ }^{2}$ C. E. DeTar and J. H. Weis, Phys. Rev. D $\underline{4}, 3141$ (1971).

${ }^{3}$ C. E. Jones, F. E. Low, and J. E. Young, Phys. Rev. D $\underline{6}, 640$ (1972).

${ }^{4}$ N. F. Bali, G. F. Chew, and A. Pignotti, Phys. Rev. 163,1572 (1967).

${ }^{5}$ M. N. Misheloff, Phys. Rev. 184, 1732 (1969).

${ }^{6}$ Early work on this form of the double-Regge vertex, including discussions of models, may be found in the following references: I. T. Drummond, Phys. Rev. 176,
2003 (1968); W. J. Zakrzewski, Nuovo Cimento 60A, 263 (1969); I. T. Drummond, P. V. Landshoff, and W. J. Zakrzewski, Nucl. Phys. B11, 383 (1969). The relevance of this form to complex helicity was originally discussed by P. Goddard and A. R. White, Nuovo Cimento 1A, 645 (1971).

${ }^{7}$ Another way of expressing the fact that there must be two terms is that the analytic structure in the variable $\kappa$ of the two terms is different.

${ }^{8}$ H. D. I. Abarbanel and A. Schwimmer, Phys. Rev. D $\underline{6}$, 3018 (1972). This paper also suggests the likelihood of two terms in the helicity-pole limit of the five-point function where $s \rightarrow \infty$ with $s_{1}, s_{2}, t_{1}$, and $t_{2}$ fixed. 